\title{
Levantamento de resíduos de laboratórios, propostas de atividades experimentais e ações com foco em Química Verde
}

Vanessa Pagno

vanessapagn@gmail.com

orcid.org/0000-0002-9885-3356 Universidade Federal da Fronteir
(UFFS), Realeza, Paraná, Brasil

André Salapata

andresalapata2@gmail.com Universidade Federal da Fronteira Sul(UFFS), Realeza, Paraná, Brasil

Edinéia Paula Sartori Schmitz edineia.schmitz@gmail.com Universidade Federal da Fronteira Su (UFFS), Realeza, Paraná, Brasil

Liziara da Costa Cabrera liziara.cabrera@uffs.edu.br

orcid.org/0000-0002-6334-0817

Urcid.org/0000-002-6334-0817 (UFFS), Realeza, Paraná, Brasil

\section{RESUMO}

Partindo da necessidade de discutir questões ligadas à Química Verde no Ensino Superior, este trabalho tem por objetivo mostrar os impactos dos resíduos químicos gerados no desenvolvimento de atividades práticas do curso de Química - Licenciatura, da Universidade Federal da Fronteira Sul (UFFS), Campus Realeza, Paraná, e propor ações para reduzir estes impactos. Para o desenvolvimento da pesquisa foram realizados levantamentos dos reagentes utilizados nas aulas experimentais do curso. Assim, foram identificados os reagentes mais tóxicos e mais utilizados e mais utilizados, descritos nos roteiros de aulas experimentais, nos períodos de 2014 e 2015. Com base nos dados levantados obteve-se um panorama dos resíduos gerados nas aulas experimentais e problematizou-se o assunto no meio acadêmico. A partir dos resultados obtidos e da problematização realizada foram elaboradas propostas de ações voltadas para a Química Verde, como uma nova forma de classificação da toxicidade dos reagentes, minimizando a quantidade de reagentes utilizados nas aulas experimentais, bem como práticas de ações sustentáveis e de educação ambiental.

PALAVRAS-CHAVE: Aulas Práticas. Resíduos. Química Verde. 


\section{INTRODUÇÃO}

A Química está presente em nosso dia-a-dia, nos diversos processos de produção, sujeitando-se à liberação de resíduos, que podem ser, muitas vezes, tóxicos e poluidores do meio ambiente (SILVA; LACERDA; JONES JUNIOR, 2005, PRADO, 2003). Em decorrência disso, houve um aumento na busca por ações que proporcionem a sustentabilidade, visando à manutenção da vida e dos recursos naturais, em todo o planeta. Essas ações surgem por parte de várias instituições governamentais, não governamentais e, até mesmo, nas Instituições de Ensino Superior (IES) (ZANDONAI et al., 2014).

Considerando que as preocupações com a gestão ambiental atingiram também as IES, surge a importância de propor ações que visem qualificar e conscientizar os graduandos quanto às questões de preservação ambiental. É importante que as Universidades passem a incorporar os princípios e práticas de sustentabilidade, dando início a processos de conscientização dentro da comunidade universitária o que é possível com decisões fundamentais como: planejar, treinar e desenvolver práticas que promovam a sustentabilidade (TAUCHEN; BRANDLI, 2006, BRASIL, 2016).

Diante disso, surge a necessidade de discutir questões ligadas às práticas sustentáveis em cursos de Química, voltando-se para as questões de Química Verde e Educação Ambiental. As discussões sobre o desenvolvimento sustentável e Química Verde foram introduzidas no Brasil, mais especificamente no final dos anos 1990, com a preocupação do desenvolvimento de novos processos químicos e tecnologias que não gerassem poluição, sendo esse, um grande desafio atualmente (PRADO, 2003, SOUZA; RIBEIRO, 2013). Desta maneira, a Química Verde defende a ideia de reduzir resíduos, especialmente os tóxicos; reduzir recursos; optar por fontes renováveis; e reduzir o consumo de energia, considerando os princípios da sustentabilidade (BAIRD; CANN, 2011, PRADO, 2003). Com estas medidas busca-se pela redução da produção de resíduos químicos tóxicos e, consequentemente, uma redução de gastos com o tratamento destes resíduos.

Baseada em doze princípios, a Química Verde se propõe a trazer ações que minimizem os impactos ambientais, visto que ela tem o caráter de prevenir a poluição gerada por atividades ligadas à química, por isso, busca-se por processos e/ou metodologias que produzam o mínimo de materiais tóxicos ou inflamáveis possíveis, reduzindo os riscos e impactos ao meio ambiente, assim como a diminuição dos gastos com o tratamento destes resíduos (SILVA; LACERDA; JONES JUNIOR, 2005, 2005) que é, hoje, motivo de grandes investimentos por parte de indústrias e IES.

Embora os laboratórios das universidades sejam utilizados para atividades como aulas experimentais, desenvolvimento de projetos de pesquisa e de extensão e trabalhos de conclusão de curso, estas atividades geram uma quantidade considerável de resíduos. Portanto, é necessária a discussão e implantação de ações menos poluentes e que tragam aos estudantes um aprendizado voltado para a preservação do meio ambiente, redução de resíduos e sustentabilidade. Uma das normas que regulamenta como deve ser realizada a identificação e classificação dos resíduos é a NBR 10004, criada em 05 de maio de 2004, que caracteriza os resíduos em: classe I, perigos; e classe II, não perigosos (NBR 10004, 2004). De acordo com esta norma, os resíduos caracterizados como perigosos são aqueles que podem apresentar riscos à saúde pública (podendo causar doenças, mortalidade, ou mesmo riscos ao meio ambiente). Já a classificação de resíduos não perigosos, considerase: classe II A, não inertes, aqueles resíduos que apresentam biodegradabilidade, combustibilidade, ou que sejam solúveis em água e que acabam gerando certa toxicidade ao meio ambiente; e classe II $B$, inertes, resíduos que não apresentam solubilidade em água e que não apresentam toxicidade ao meio ambiente (BRASIL, 2004).

Outra norma que também foi criada para auxiliar na classificação para o tratamento de resíduos, é dada pela resolução da Diretoria Colegiada - RDC 306, de 07 de dezembro de 2004, da Agência Nacional de Vigilância Sanitária, ANVISA (BRASIL, 2006). De acordo com esta norma, os resíduos podem ser classificados como: Grupo A1, que compreende 
resíduos de fabricação de produtos biológicos, microrganismos, com exceção dos hemoderivados; Grupo A2, A3, A4, e A5, compreendem material biológico, como peças anatômicas, órgãos, tecidos, perfurocortantes, dentre outros; Grupo $B$, que compreende produtos farmacêuticos e cosméticos; Grupo $C$, que compreende os rejeitos radioativos ou contaminados com radionuclídeos; Grupo D, que compreende resíduos que não apresentam riscos biológicos ou químicos, que possam ser reciclados ou reutilizados; e Grupo E, que compreende rejeitos perfurocortantes como navalhas, agulhas, dentre outros (BRASIL, 2006).

Com base nessas normas, toda e qualquer atividade que gere resíduos deve possuir um gerenciamento correto dos mesmos. Diante disso, a Universidade Federal da Fronteira Sul, Campus Realeza, Paraná, preocupada com os riscos apresentados pelos resíduos gerados nas aulas experimentais, elaborou, por meio da Coordenação Adjunta dos Laboratórios, um Plano de Gerenciamento de resíduos. Este plano segue a Resolução 306 da ANVISA de 2004 (BRASIL, 2015).

Como a UFFS não tem seu próprio sistema para tratar os resíduos gerados, os mesmos são recolhidos por uma empresa externa, mediante contrato de prestação de serviços especializados em tratamento e destinação final de resíduos. Esta empresa externa recolhe os resíduos divididos em categorias (segundo as normas da ANVISA). Alguns destes resíduos são incinerados, outros autoclavados, a depender de suas características e conforme o sistema de tratamento disponível na sede da empresa contratada. Para cada lote de resíduos coletado e tratado pela empresa prestadora de serviços, é emitido um laudo de destinação correta destes resíduos, comprovando que os mesmos foram tratados adequadamente. $\mathrm{O}$ tratamento dos resíduos gerados nos laboratórios acarreta um custo elevado à universidade, uma vez que cada quilograma de resíduo entregue para tratamento custa, atualmente, em torno de onze reais.

Tendo em vista as questões de geração de resíduos e de custo para tratamento, neste trabalho realizou-se um levantamento dos resíduos laboratoriais gerados na Instituição, problematizou-se o impacto ambiental dos mesmos e a necessidade de assumir ações de sustentabilidade ambiental dentro da Universidade. Isto foi possível por meio de análises qualitativas e quantitativas dos reagentes utilizados nos experimentos e dos resíduos gerados nos laboratórios da Universidade. A partir disso, propôs-se uma nova classificação da toxicidade dos reagentes, bem como ações e práticas voltadas para a Química Verde.

\section{METODOLOGIA}

Com base nos reagentes utilizados, identificaram-se os mais tóxicos e os mais utilizados nas aulas práticas do curso, com base nas análises qualitativas e quantitativas dos roteiros de aulas experimentais desenvolvidas pelos professores dos componetes curriculares do curso de Química - Licenciatura nos anos de 2014 e de 2015. A partir disso, iniciou-se a etapa de tabulação e construção de gráficos dos dados obtidos a fim de demonstrar a geração de resíduos químicos, por ano e por componente curricular.

É importante salientar que as quantidades de reagentes utilizadas são uma estimativa, sendo que não se considerou a concentração dos reagentes devido à falta desta informação em alguns dos roteiros de aulas e pelo fato de a entrega de resíduos dos laboratórios ocorrerem numa proporção 1:1, em que 1 litro (L) de resíduos equivale a 1 quilo $(\mathrm{kg})$, conforme definido em contrato. Com base nos dados coletados pode-se obter uma estimativa do quantitativo dos resíduos gerados nas aulas experimentais, apenas analisando a quantidade geral dos reagentes utilizados.

Na segunda etapa do trabalho, realizou-se a classificação dos reagentes de acordo com sua toxicidade, a partir da montagem de um fluxograma que permitisse classificar determinado roteiro de aula prática de acordo com a toxicidade dos reagentes utilizados, propondo assim, discussões acerca das práticas realizadas no curso de Química - 
Licenciatura. Foram ainda elencadas ações menos poluentes, que enfatizam a importância da diminuição dos resíduos laboratoriais nas atividades práticas.

\section{RESULTADOS E DISCUSSÕES}

\section{ANÁLISE DOS RESÍDUOS ENTREGUES PARA TRATAMENTO}

De acordo com a ANVISA, os resíduos químicos gerados nos laboratórios podem ser classificados como: A1; A2; A3; A4; A5; B; C; D; e E. No entanto, no Campus Realeza, com base no contrato de coleta, os resíduos são separados em três categorias, sendo compreendidos: (I) grupo A1, A4, A5 e E; (II) grupo B; (III) grupo A3 e A5, conforme Tabela 1.

Analisando os resíduos desses grupos de acordo com o que é gerado no curso de Química - Licenciatura, percebe-se que: o grupo $\mathrm{E}$, próprio para rejeitos perfuro cortantes, não houve a geração destes resíduos nas aulas de química do curso estudado. $O$ grupo $A$, compreende alguns resíduos gerados nas aulas experimentais de Química, como luvas, papel filtro contaminado, dentre outros materiais orgânicos. Já o grupo B compreende os resíduos contendo substâncias químicas, que são os mais utilizados nas aulas experimentais do curso.

Tabela 1 - Resíduos entregues para tratamento, em 2014 e 2015

\begin{tabular}{cccc}
\hline Ano & $\begin{array}{c}\text { Grupo A1, A4, A5 e E } \\
(\mathrm{kg})\end{array}$ & Grupo B (kg) & Grupo A3 (kg) \\
\hline 2014 & 153,5 & 275,4 & 6,40 \\
\hline 2015 & 105,7 & 254,3 & 11,50 \\
\hline
\end{tabular}

Fonte: Autoria própria (2017)

Desta maneira, pode-se analisar que foram entregues 435,5 kg de resíduos em 2014, e um total de $371,5 \mathrm{~kg}$ de resíduos em 2015. Assim, no ano de 2014, houve uma maior geração de resíduos. Um dos motivos para esta diferença, observados por meio de análise quantitativa dos roteiros de aulas experimentais, foi que em 2014 foram ministradas mais aulas experimentais no curso de Química do que no ano de 2015. Cabe ainda ressaltar que parte desses resíduos é gerada também pelos outros cursos ministrados na Universidade, pois estes dados tratam da geração total de resíduos dos laboratórios da UFFS nos anos citados.

Assim, considerando os dados gerais e totais, dos resíduos destinados ao tratamento por empresa especializada, não foi possível obter dados exatos da quantidade de resíduos produzidos nas aulas experimentais apenas do curso de Química - Licenciatura, pois os resíduos de todos os cursos são entregues de forma conjunta, sendo possível apenas observarmos que as quantidades de resíduos destinadas variaram de um ano para outro e que no ano de 2015 a quantidade gerada foi menor do que em 2014.

\section{ANÁLISES DOS RESÍDUOS GERADOS NAS AULAS EXPERIMENTAIS DO CURSO DE QUÍMICA - LICENCIATURA}

Como forma de quantificar os resíduos gerados nos laboratórios, utilizou-se a identificação dos reagentes utilizados nas aulas experimentais do curso de Química Licenciatura, no período de 2014 a 2015. Na Figura 1 estão elencadas as 78 aulas experimentais ministradas em 2014 e as 68 aulas experimentais ministradas no ano de 2015, por componente curricular ofertado. 
Todos os roteiros de aulas experimentais realizados foram analisados e obteve-se um panorama dos reagentes utilizados, quais destes reagentes foram utilizados em maior quantidade e em quais componentes curriculares, bem como suas quantidades. Considerando que todos os reagentes utilizados nas aulas experimentais, transformaramse em resíduos, pode-se calcular a massa de reagentes consumidos por estudante e o custo de destinação destes reagentes por aluno, tendo como base o valor de destinação de $\mathrm{R} \$$ 11,00 para cada kg de resíduo coletado.

Desta maneira, percebeu-se que houve um aumento significativo no número de aulas práticas da área de Analíticas, sendo que, em 2014, foram realizados 24 experimentos, com um número 68 de reagentes utilizados, num total de 48,20 kg de resíduos gerados. Já em 2015, foram 32 experimentos realizados, com um número 67 de reagentes utilizados, num total de 59,79 kg de resíduos gerados. Além do componente curricular de Físico-Química Experimental, que teve uma geração de $53,3 \mathrm{~kg}$ de reagentes, tendo sido ofertada para 18 alunos com um custo de aproximadamente 32,56 reais por aluno.

Figura 1 - Aulas experimentais ministradas em 2014 e 2015

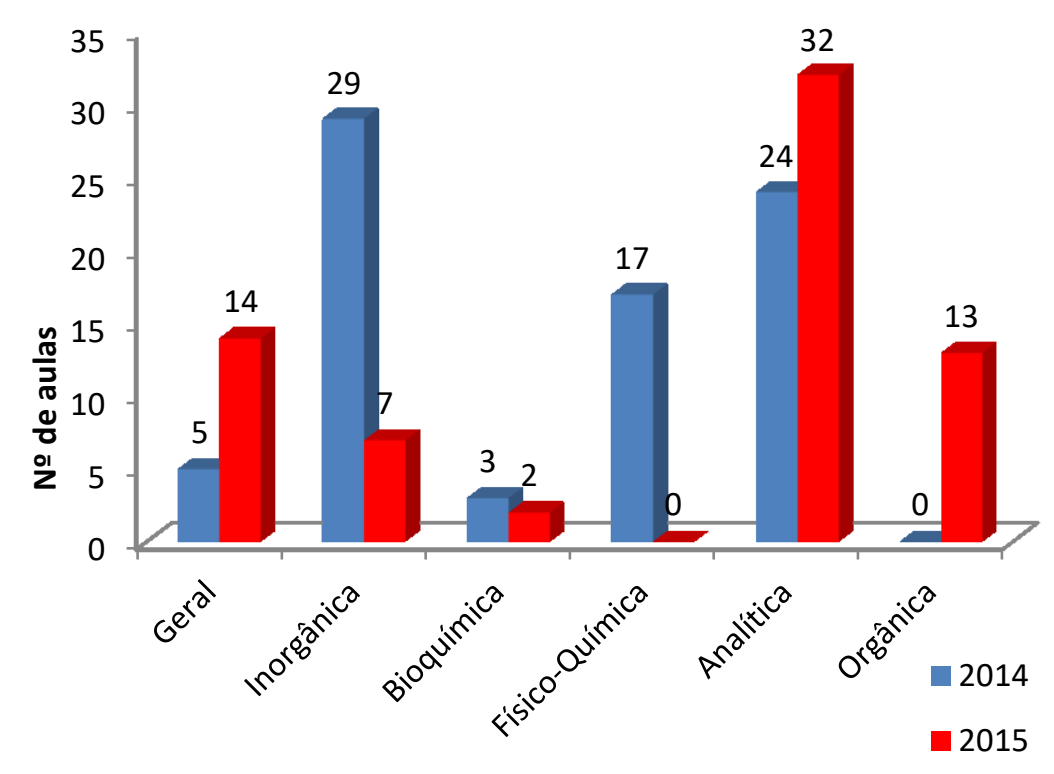

Fonte: Autoria própria (2017)

Com base nos dados, nota-se que houve uma variedade grande de reagentes utilizados num período de dois anos, no curso. Uma vez que, estes reagentes, quando utilizados, tornam-se resíduos, trazem um elevado custo para a Universidade, tanto pela aquisição, quanto pela destinação dos mesmos, pois estes resíduos gerados são enviados para tratamento realizado pela empresa externa.

O custo de tratamento dos resíduos, conforme já mencionado, encontra-se em torno de 11 reais por $\mathrm{kg}$ de resíduo entregue. Portanto, considerando que os reagentes utilizados no ano de 2014 foram transformados em resíduos, tem-se um total de 129,07 kg de reagentes utilizados, assim, seu tratamento custou em torno de $\mathrm{R} \$ 1.420,00$. Já em 2015, quando se utilizou uma quantidade de $120,58 \mathrm{~kg}$ de reagentes, o custo de tratamento foi de, aproximadamente, $\mathrm{R} \$ 1.326,00$.

\section{REAGENTES MAIS UTILIZADOS NAS AULAS EXPERIMENTAIS}

Com base na análise dos roteiros de aulas experimentais, do curso de Química Licenciatura, da UFFS, também se identificou os 10 reagentes mais utilizados em termos 
de quantidades. Na Figura 2 podem-se observar os resultados desta análise, para o ano de 2014, onde estão elencados os 10 reagentes mais utilizados, sendo eles: ácido clorídrico; hidróxido de sódio; etanol; sulfato de cobre; cloreto de amônio; ácido acético; bissulfato de sódio; etanol; sulfato de cobre; cloreto de amônio; e ácido sulfúrico.

Figura 2 - Reagentes mais utilizados, no curso de Química - Licenciatura, no ano de 2014, valores em $\mathrm{kg}$

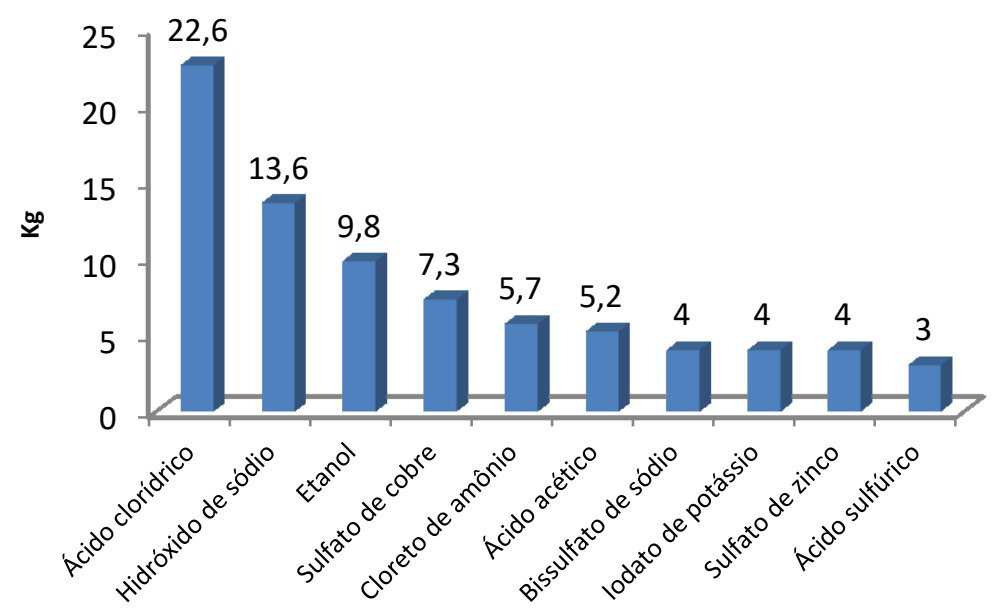

Fonte: Autoria própria (2017)

Em 2015, os 10 reagentes elencados como os mais utilizados nas aulas experimentais, são indicados na Figura 3, sendo eles: ácido clorídrico; nitrato de prata; hidróxido de sódio; etanol; metanol; tiocianato de amônio; carbonato de cálcio; diclorometano; tiossulfato de sódio; e ácido sulfúrico.

Figura 3 - Reagentes mais utilizados, nas aulas experimentais, no ano de 2015 , valores em $\mathrm{kg}$

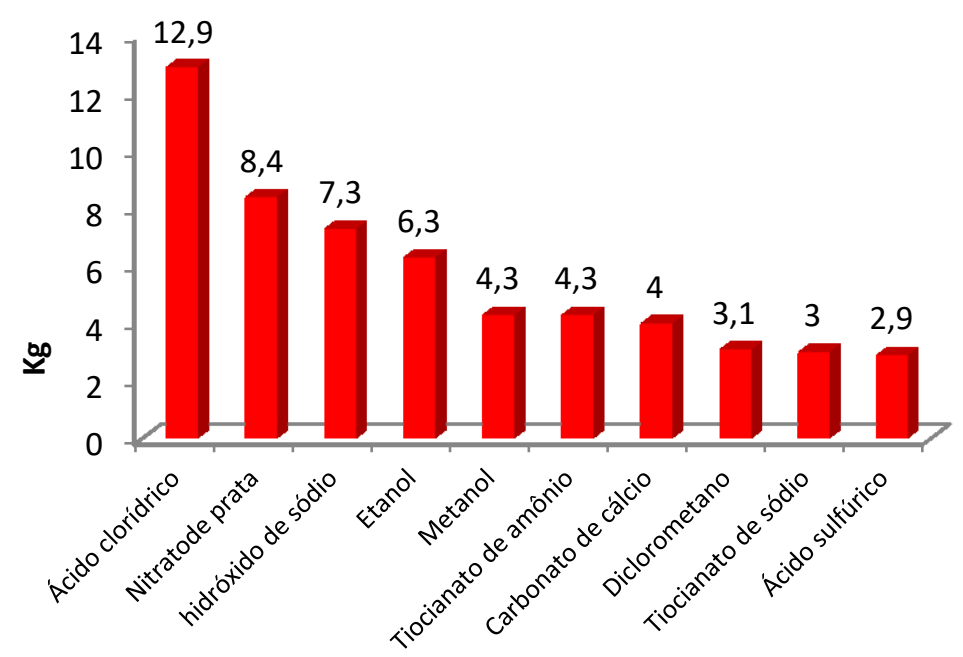

Fonte: Autoria própria (2017)

Com base nesses dados percebe-se que o ácido clorídrico foi o reagente mais utilizado nas aulas experimentais do curso de Química - Licenciatura, no período de análise, sendo que em 2014, foram utilizados 22,6 kg deste reagente e em 2015 foram utilizados 12,9 kg, pode-se perceber também que no ano de 2014 ocorreu um maior consumo deste reagente. 


\section{PROPOSTAS DE AÇÕES SUSTENTÁVEIS, COM FOCO NA QUÍMICA VERDE}

Com a identificação dos custos no tratamento dos resíduos gerados nas aulas experimentais do curso de Química- Licenciatura, e a análise dos reagentes utilizados e suas quantidades, também se realizou uma análise de toxicidade dos reagentes utilizados no período de 2014 a 2015, com base nas Fichas de Informações de Segurança dos Produtos Químicos (FISPQ's).

Uma vez que, na literatura não existe um parâmetro de toxicidade comum aos reagentes químicos utilizados na Universidade neste trabalho utilizou-se a Dose letal ( $D_{50}$ ), via oral, em ratos como um dado para classificar a maioria dos reagentes. Desta maneira, foram construídas categorias de toxicidade para classificar uma lista extensa de reagentes, todos utilizados nas aulas práticas do curso de Química - Licenciatura, demonstrando o risco ambiental envolvido nestas práticas e a necessidade de uma ação efetiva sobre as mesmas. Esta categorização se deu com base na identificação da $D_{50}$ dos reagentes utilizados e posterior divisão em quatro classes, sendo que quanto menor a Dose Letal, mais tóxico o reagente se torna.

Os reagentes identificados como sendo de elevada toxicidade foram denominados como Classe I, de acordo com sua DL50 (via oral - ratos), estão elencados na Figura 4, e apresentam um valor entre 30 e $350 \mathrm{mg} / \mathrm{kg}$. Já as demais classes elencadas a partir dos reagentes utilizados nos laboratórios da UFFS, Campus Realeza, e de suas DL50, foram categorizadas como: Classe II - DL50 de 350 a 2000 mg/kg - Toxicidade Regular, como podese analisar na Figura 5; Classe III -DL50 de 2000 a 5000 mg/kg - Toxicidade Média, como pode-se analisar na Figura 6; e Classe IV - DL50 acima de 5000 mg/kg - Toxicidade Baixa, na Figura 7.

Observa-se que, para a categoria denominada como "Classe I" - Toxicidade Elevada encontram-se 23 reagentes utilizados nas atividades práticas, fato que demonstra o risco a que estão expostos os estudantes e docentes, bem como o meio ambiente, visto que todos os reagentes transformam-se em resíduos, potencialmente poluidores e que necessitam de tratamento adequado. Práticas que visem à redução, ou mesmo eliminação do uso destes reagentes, são necessárias para uma visão mais voltada para a Química Verde. 
Figura4 - Categoria 1, DL50 de $30 \mathrm{mg} / \mathrm{kg}$ a $350 \mathrm{mg} / \mathrm{kg}$ - Toxicidade elevada

\section{Classe I - DL50 de 30 a 350 mg/Kg}

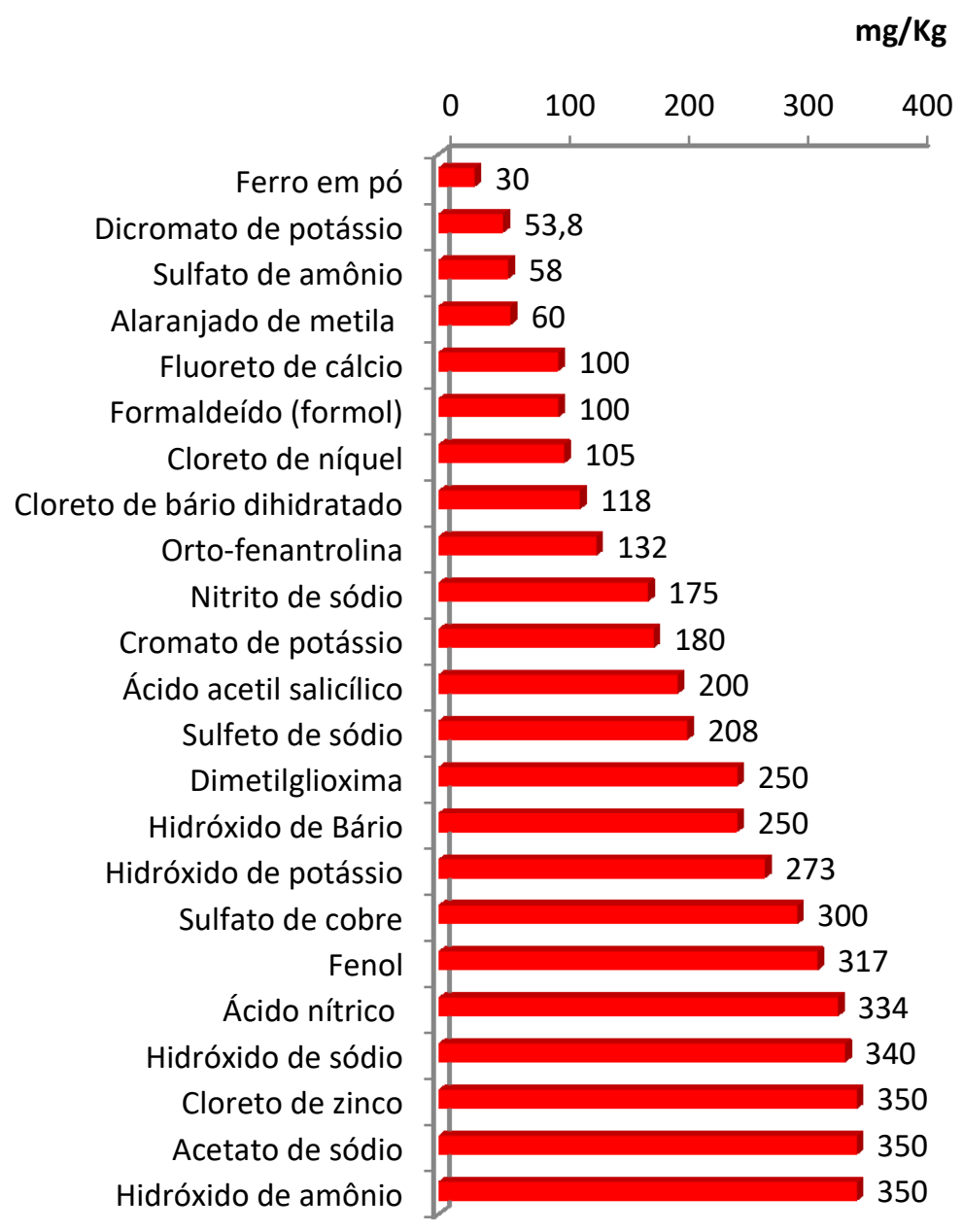

Fonte: Autoria própria (2017)

Um reagente tóxico, destacado na classificação, é o alaranjado de metila, que chama a atenção por ser um indicador bastante utilizado nas aulas experimentais do curso de Química - Licenciatura, principalmente nas aulas de Analítica. Os dados desta classificação conferem se com a literatura, pois de acordo com Cadorin (2009) este reagente é tóxico por inalação, sendo que, se resíduos contendo este composto forem despejados no meio ambiente causam diversos danos ao ecossistema (CADORIN, 2009). Em meios aquáticos poderá causar poluição estética e de eutrofização - reduzindo a penetração da luz na água, o que diminui o oxigênio dessa água podendo colocar os microrganismos presentes nessa água em risco, podendo também, formar subprodutos perigosos por meio de reações como de oxidação, hidrólise, dentre outras. 
Figura 5 - Categoria 2, DL50 de $350 \mathrm{mg} / \mathrm{kg}$ a $2000 \mathrm{mg} / \mathrm{kg}$ - Toxicidade Regular

\section{Casse II - DL50 de 350 a 2000 mg/Kg}

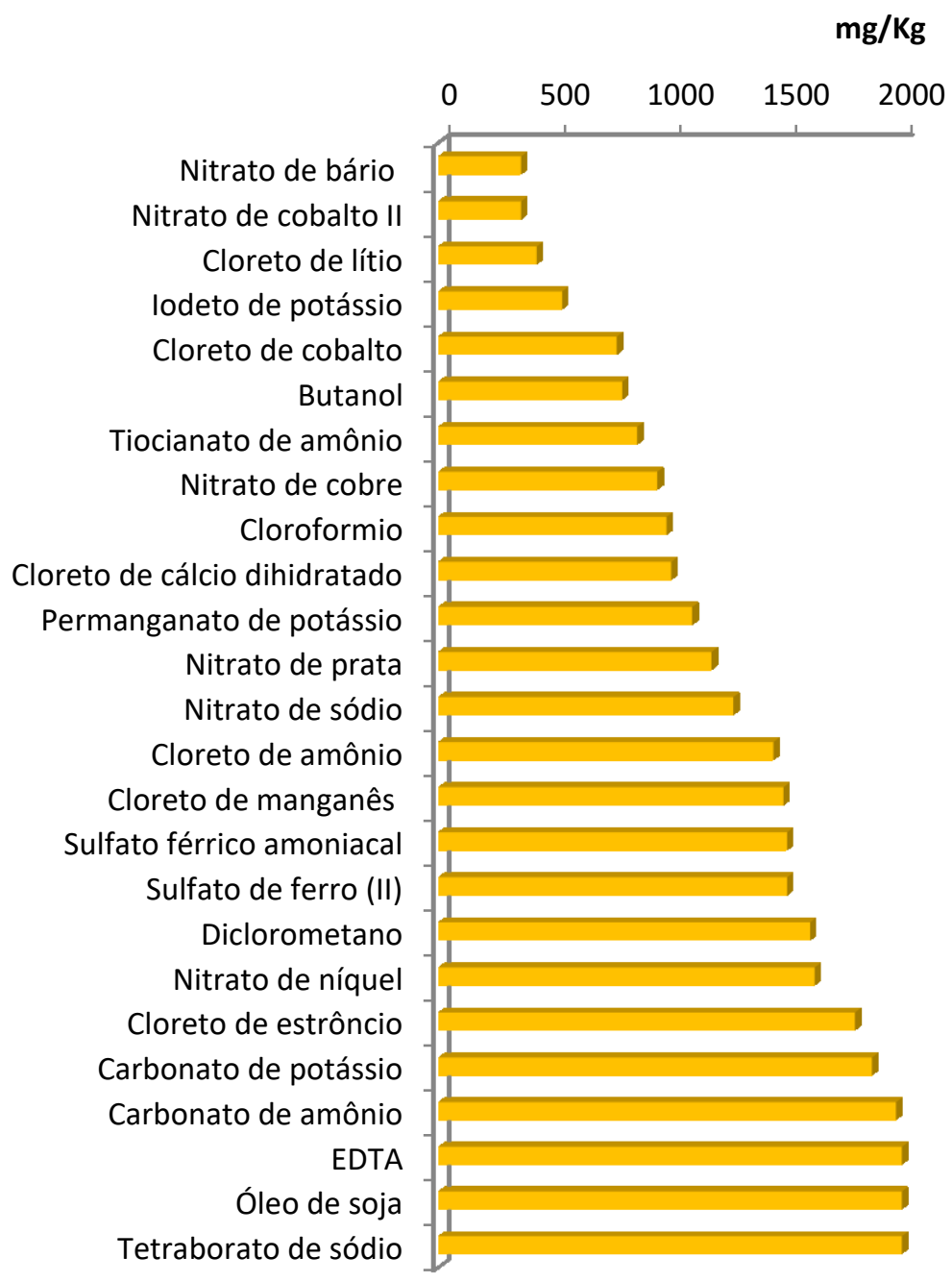

Fonte: Autoria própria (2017)

Com relação à "Classe II", ora denominada como Toxicidade Regular, Figura 5, encontram-se uma gama de reagentes que são muito comuns quanto ao uso em atividades de laboratório. Dentre estes, destaca-se um solvente bastante utilizado em aulas experimentais, o ácido etilenodiaminotetracético (EDTA), que possui uma DL50 considerada regular e que também apresenta risco de contaminação ao meio ambiente. Uma vez que, se descartado incorretamente, este reagente pode formar complexos estáveis com íons metálicos do meio, sendo hoje considerado como um poluente emergente (ANDRADE et al., 2016), pois pode causar danos ao meio ambiente.

Diante disso, há a necessidade de repensar as nossas ações, métodos e materiais utilizados, em aulas experimentais, bem como a nossa visão enquanto educadores e formadores de cidadãos. O estudo das FISPQ's dos reagentes mais utilizados nas aulas experimentais da instituição propiciou essas discussões importantes sobre sustentabilidade e educação ambiental e conduziu a estes questionamentos. Além disso, essa discussão possibilita que os acadêmicos conheçam melhor a FISPQ's dos reagentes, 
discutindo aspectos de saúde, e redobrando cuidados como a importância do uso de EPIs em aulas práticas.

Figura 6 - Categoria 3, DL50 de 2000 mg/kg a 5000 mg/kg - Toxicidade Média

\section{Classe III - DL50 de 2000 a 5000 $\mathrm{mg} / \mathrm{Kg}$}

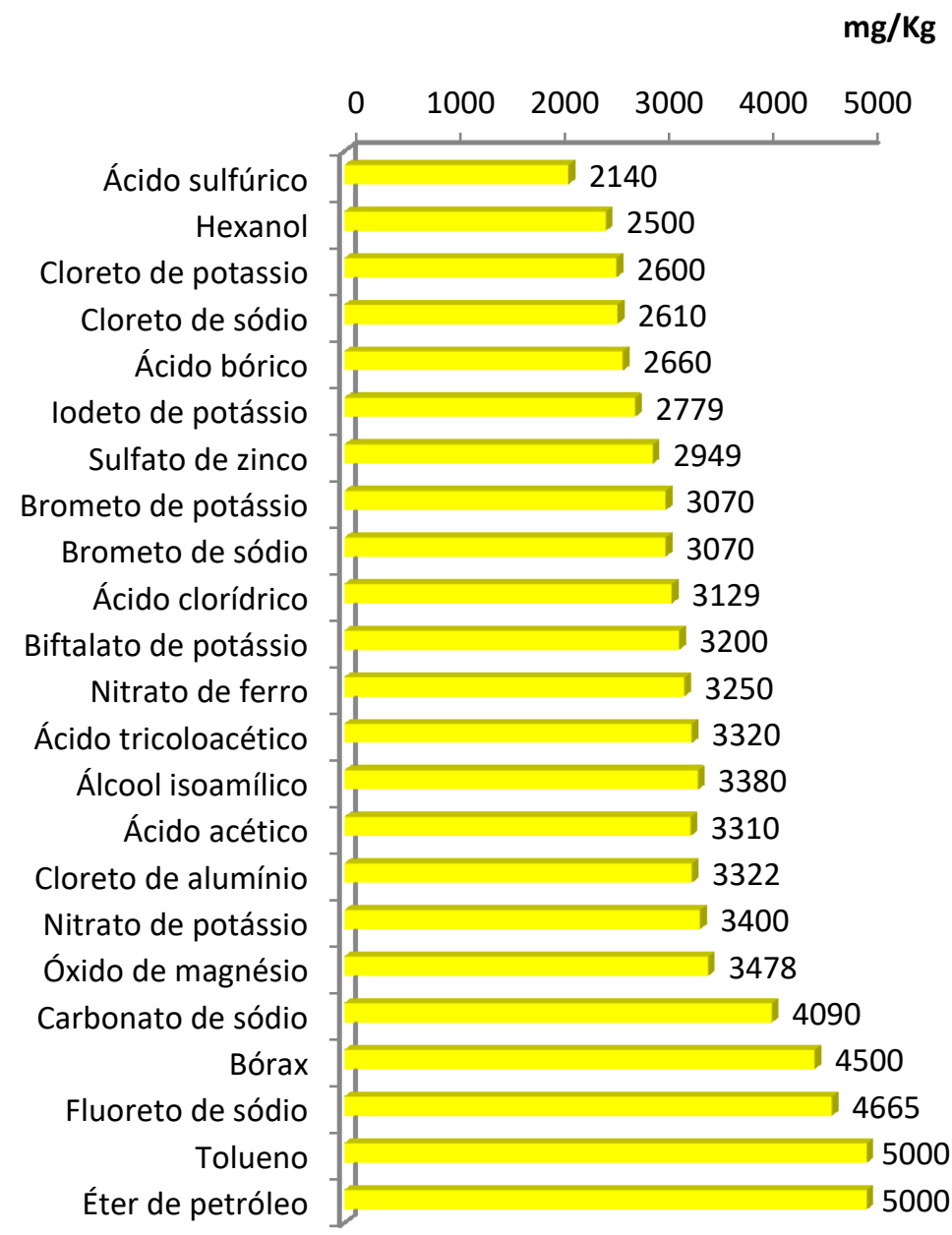

Fonte: Autoria própria (2017)

Quanto à "Classe III" (Figura 6) e "Classe IV "(Figura 7), Toxicidades média e baixa, respectivamente, estão distribuídos os demais reagentes utilizados nas atividades práticas. Vale destacar que, mesmo os reagentes listados na "Classe IV", considerada aqui de baixa toxicidade, ainda assim existe uma toxicidade, ou seja, a contaminação e o prejuízo ambientais podem ocorrer da mesma forma, se estes produtos não forem devidamente descartados e tratados. 
Figura 7 - Categoria 4, DL50 $>5000 \mathrm{mg} / \mathrm{kg}$ - Toxicidade Baixa

\section{Classe IV - DL50 > 5000 mg/Kg}

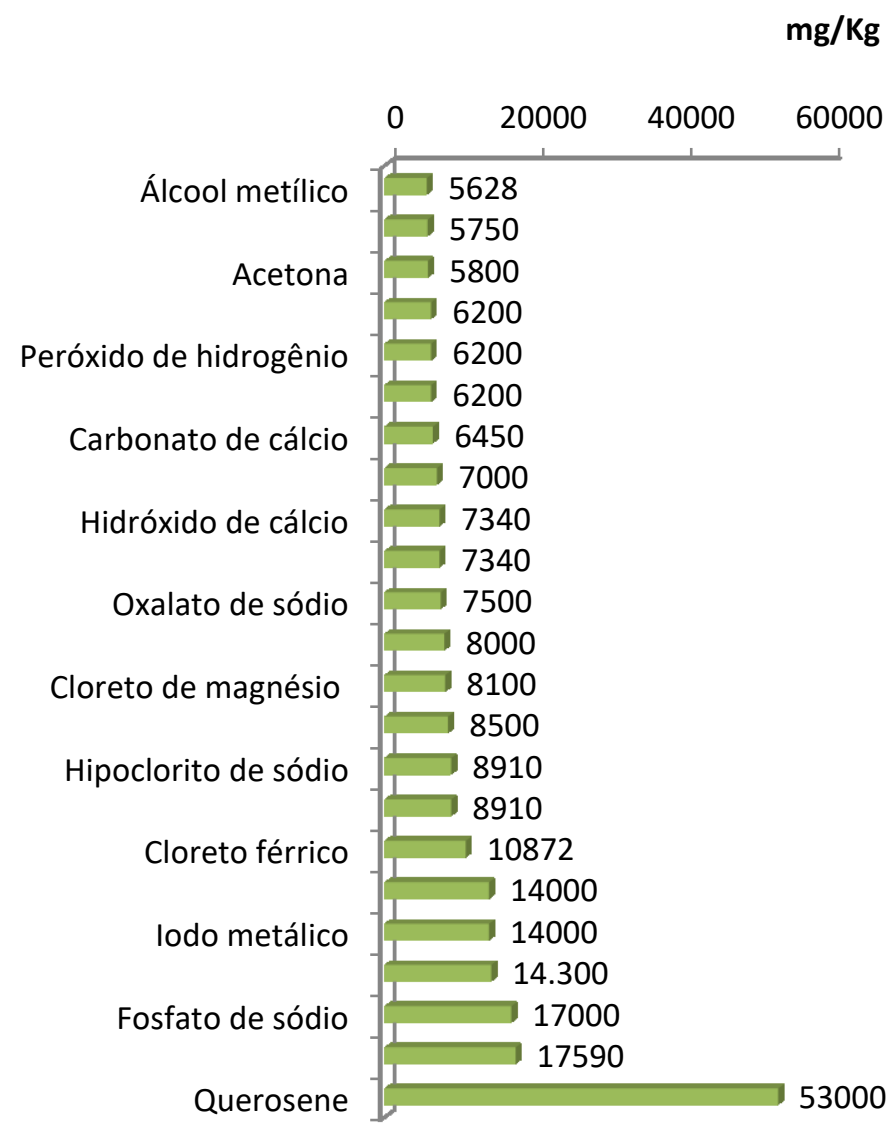

Fonte: Autoria própria (2017)

Partindo destas categorias, foram levantadas algumas ações sustentáveis para minimizar a quantidade de resíduos gerados após as aulas experimentais do curso de Química - Licenciatura. Uma destas propostas é indicada no fluxograma apresentado na Figura 8 em que se propõe uma classificação dos reagentes por classes de toxicidade comparativa, com base nas $\mathrm{DL}_{50}$ (via oral - em ratos) encontradas para os reagentes já utilizados nas aulas experimentais do curso.

Com base neste fluxograma, os professores dos componentes curriculares experimentais, do curso de Química, podem ter uma identificação de como suas aulas se tornam mais ou menos poluentes, em termos de toxicidade ao meio ambiente, caso estes resíduos não apresentem a destinação correta, por exemplo. Neste ponto considerou-se apenas a toxicidade ao meio ambiente, pois, considera-se que o contato dos alunos e dos professores com estes reagentes se dá de maneira segura - sendo que ambos utilizam equipamentos de segurança (luva, óculos, jaleco, dentre outros) e que todos os reagentes voláteis sejam manipulados na capela de exaustão de gases. 
Figura 8 - Fluxograma de toxicidade de acordo com a DL50 de cada reagente

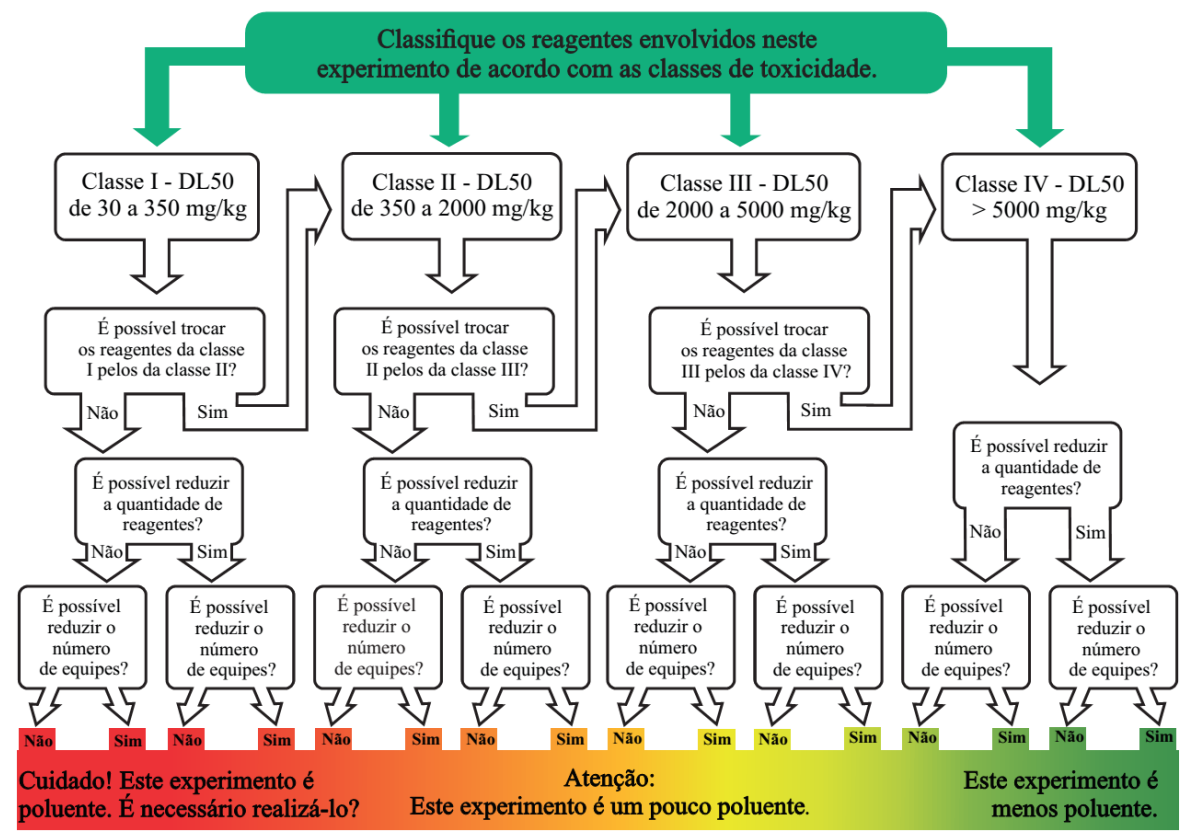

Fonte: Autoria própria (2017)

Com base neste fluxograma, os professores podem ter uma noção da toxicidade de cada reagente utilizado em suas aulas experimentais. Neste sentido, pode-se optar pela troca de um reagente mais tóxico, por outro menos tóxico, ou reduzir as quantidades utilizadas dos reagentes mais tóxicos.

Neste sentido, pensando em reduzir os resíduos gerados nas aulas experimentais, realizou-se um exercício no sentido de elencar algumas atitudes que podem reduzir em muito os reagentes utilizados, um exemplo deste exercício é apresentado para o componente curricular de Química Analítica, onde se tem como opção a redução de reagentes utilizados, uma vez que nestas disciplinas, geralmente, utiliza-se uma grande quantidade e grande variedade de reagentes, bem como, realizam-se as prática em duplicatas e/ou triplicatas.

Assim, propõe-se que no componente de Química Analítica, realize-se, apenas a identificação dos cátions dos grupos III, IV e V, pois os alunos podem obter o aprendizado sobre a identificação de todos os dos cátions com estas práticas, evitando a identificação dos grupos I e II, que necessitam de reagentes altamente tóxicos ao meio ambiente, como o mercúrio e o chumbo. Desta maneira, propõem-se apenas a explicação teórica desta prática envolvendo os grupos I e II e a redução dos reagentes dos outros grupos, ou mesmo uma redução no número de grupos por turma a realizar a prática.

Outra ação menos poluente que pode ser pensada como propósito de redução e minimização dos resíduos gerados na Universidade e, consequentemente, redução dos custos deste tratamento, é o tratamento dos resíduos aquosos durante as próprias aulas práticas, pelos estudantes. Este tratamento pode ser realizado após as aulas experimentais - recomenda-se que seja realizado ao final do componente curricular - em que os alunos podem fazer a precipitação dos resíduos presentes nas soluções aquosas, com posterior filtração e neutralização dos mesmos, utilizando para tal, um reagente alcalino, como o hidróxido de sódio ou de cálcio. Com isto, os resíduos que serão entregues para tratamento serão minimizados, pois a parte líquida, já tratada e separada dos seus precipitados, pode ser descartada até mesmo na pia (conferindo-se o pH). Desta maneira, reduz-se o volume a ser tratado por empresa externa e também os impactos ambientais causados. Já que a maioria dos resíduos entregues para tratamento são soluções. 
De acordo com a literatura, os resíduos contendo sais de cloreto de bário e cálcio podem ser tratados com a adição de $10 \%$ de sulfato de sódio, com aquecimento desta mistura e evaporação do sobrenadante. Com esta prática, tem-se ao final o cloreto de sódio que não é tóxico ao meio ambiente e o sulfato de bário que pode ser armazenado para posterior tratamento, mas que, no entanto, se encontra num volume menor (SANTOS et al., 2011). Já para resíduos contendo sais de cloreto de magnésio, o tratamento antecipado pode ser dado com a adição de hidróxido de sódio, formando um precipitado de hidróxido de magnésio. Deixando a solução em repouso, esta pode ser filtrada posteriormente e neutralizada com ácido clorídrico e pode ser descartada. Já o hidróxido de magnésio formado nessa reação pode ser reaproveitado em outras práticas (SANTOS et al., 2011).

Com base nessas ações, os acadêmicos estarão também aprendendo sobre reações de neutralização, precipitação, dentre outras e, ao mesmo tempo, estarão sendo conscientizados e alertados para as toxicidades dos reagentes, uma vez que o professor do componente curricular pode abordar diversos conceitos ligados à redução de resíduos em aulas experimentais e ações voltadas para a Química Verde. Neste sentido, os professores podem explorar conceitos ligados à Química verde e educação ambiental em sala de aula, para conscientizar os alunos de que os resíduos gerados no laboratório também precisam ser tratados e descartados corretamente, atendo-se para os danos que estes podem causar ao meio ambiente. Essa proposta visa formar profissionais capacitados e responsáveis pela sustentabilidade do planeta, que tenham como regra, utilizar da ciência para manter e melhorar a qualidade de vida, sendo que essa regra deve ser um dos objetivos dos cursos de Química (PRADO, 2005).

Princípios de sustentabilidade têm sido desenvolvidos nos cursos de Química, em geral, em disciplinas isoladas, uma delas é a Química Ambiental, mas poucas ações práticas ocorrem e essas em geral não são multidisciplinares (COLLINS, 2017). Nesse sentido a proposta deste trabalho permeia por várias disciplinas do curso de Química, abordando ações práticas de sustentabilidade.

$\mathrm{Na}$ literatura são encontrados trabalhos que minimizam o uso de solvente, principalmente com o foco na Química Analítica Verde (ARMENTA et al., 2017). Mas trabalhos que enfoquem aulas experimentais sustentáveis são ainda incipientes (ZANDONAl et al., 2016)

\section{CONCLUSÃO}

O levantamento dos dados sobre os usos de reagentes nas atividades práticas, desenvolvidas no curso de Química - Licenciatura, bem como dos resíduos gerados nestas atividades, durante os anos de 2014 e de 2015, possibilitou aos pesquisadores, identificar uma variedade de reagentes e que possuem toxicidade, com base na análise dos limites de $\mathrm{DL}_{50}$ (via oral em ratos) de cada produto. Confirmando que as práticas desenvolvidas, em sua maioria, não apresentam uma preocupação com os resíduos gerados.

Outro fato identificado foi a variedade de produtos utilizados, fato preocupante, visto os perigos envolvidos na mistura de substâncias, gerando resíduos de maior toxicidade. Além da variedade, identificou-se que, em termos de quantidades de resíduos gerados, mesmo sendo um curso de Química modalidade Licenciatura, a geração de resíduos é considerável, sendo perfeitamente possível projetar uma redução nestes volumes.

A redução dos volumes de resíduos gerados pode ser dada através da análise dos roteiros de aulas propostos pelos docentes, sendo que, através da proposta do Fluxograma criado durante o desenvolvimento da pesquisa, são apresentados caminhos para esta análise, todos eles levando sempre a práticas menos poluentes e que prezam pela Química Verde. 
Assim sendo, considera-se o exercício da análise dos roteiros das aulas o fator primordial na busca de ações voltadas para a Química Verde e educação ambiental, fato que nos motiva a buscar novos meios para trabalhar a experimentação no ensino da Química, minimizando a produção de resíduos e, ao mesmo tempo, conscientizando os alunos sobre o tema através de ações menos poluentes. 


\title{
Waste monitoring and proposal of activities focused on green chemistry in an undergraduate chemistry program
}

\begin{abstract}
Based on the need to discussing questions linked to Green Chemistry at Higher Education, the objective of this project was to show the impacts of chemical waste generated from practical activities developed during a Chemistry Teaching degree program, at the Realeza campus of the Federal University of FronteiraSul (UFFS), and propose actions to reduce these impacts. For this, qualitative and quantitative analyses of reagents used in chemistry experimental classes, were performed, having as basis, the identification of the most used and toxic reagents, described during experimental classes protocols, in the period from 2014 to 2015. With the results, it was obtained the panorama of waste generated during experimental classes and problematized the subject in the higher education. Thus generating the proposal actions focused on Green Chemistry which minimize to reduce the quantity of reagents used in experimental classes as well as practice sustainable actions.

KEYWORDS: Experimental classes. Waste. Green Chemistry.
\end{abstract}




\section{REFERÊNCIAS}

ALENCAR, L. H.; ALMEIDA, A. T.; MOTA, C. M. M. Sistemática proposta para seleção de fornecedores em gestão de projetos. Gestão \& Produção, São Carlos, v. 14, n. 3, p. 477-487, set./dez. 2007. Disponível em:

$<$ http://www.scielo.br/scielo.php?script=sci arttext\&pid=S0104-

530X2007000300005\&lng=pt\&nrm=iso >. Acessoem: 23 jul. 2016.

ANDRADE, C. E. O.; OlIVEIRA, A. F.; NEVES, A. A.; QUEIROZ, M. E. L. R. A new spectrophotometric method for determination of EDTA in water using its complex with Mn(III). Spectrochimia Acta - Part A: Molecular Spectroscopy. V. 168, p. 253-277, 2016. Dissponível em:

<http://www.sciencedirect.com/science/article/pii/S1386142516303262>. Acessoem: 15/06/2017.

ARMENTA, S.; ESTEVE-TURRILLAS, F. A.; GARRIGUES, S.; DE LA GUARDIA, M. Green Analytical Chemistry: The Role of Green Extraction Techniques. Comprehensive Analytical Chemistry. V.76, p. 1230-1254, 2017.

BAIRD, C.; CANN, M. Química Ambiental. 4. ed. Porto Alegre: Brookman, 2011. 844 p. Tradução: Marco Tadeu Grassi.

BRASIL. Ministério da Saúde. Agência Nacional de Vigilância Sanitária. Manual de gerenciamento de resíduos de serviços de Saúde. Brasília, 2006.

BRASIL. Universidade Federal da Fronteira Sul. Plano de Gerenciamento de resíduos de laboratórios do Campus Realeza - PR. 2015.

BRASIL. ABNT. NBR 10.004: (2004). Resíduos sólidos - Classificação. Rio de Janeiro: $77 \mathrm{p}$.

BRASIL. EPA. Universities Lend a Hand with Sustainability. Disponivel em: https://www.epa.gov/sciencematters/universities-lend-hand-sustainability. Acesso em junho de 2016.

CADORIN, B. M. Degradação do corante Alaranjado de Metila por plasma frio de descarga corona. (Trabalho de Conclusão de Curso). Universidade Federal de Santa Catarina, Química. 2009. Disponível em: <https://repositorio.ufsc.br/handle/123456789/99778>. Acesso em: 02/04/2016.

COLLINS, T. J. Review of the twenty-three year evolution of the first university course in green chemistry: teaching future leaders how to createsustainable societies. Journal of 
PRADO, G. S. A. Química verde, os desafios da química do novo milênio. Química Nova, vol.26, n.5, p. 738-744.2003. Disponível em:

$<$ http://quimicanova.sbq.org.br/imagebank/pdf/Vol26No5 738 17-

DV02190.pdf >. Acesso em: 02/03/2016.

SANTOS, V. M. L.; MEDRADO, L. S.; JÚNIOR, J. E. S.; SILVA, J. A. B. Proposta para disposição final dos resíduos químicos identificados nos laboratórios do Campus da Fazenda Experimental / UNIVASF. GEPROS. 2011. Disponível em:

$<$ http://revista.feb.unesp.br/index.php/gepros/article/view/437>. Acesso em: 02/04/2016.

SILVA, F. M.; LACERDA, P. S. B.; JONES JUNIOR, J., . Desenvolvimento sustentável e química verde. Química Nova, v. 28, n.1, p. 103-110. 2005. Disponível em: <http://professor.pucgoias.edu.br/SiteDocente/admin/arquivosUpload/3280/ma terial/23046.pdf>. Acesso em: 05/03/2016.

SOUZA, M. T. S.; RIBEIRO, H. C. M. Sustentabilidade Ambiental: uma Meta-análise da Produção Brasileira em Periódicos de Administração. RAC, Rio de Janeiro, v. 17, n. 3, art. 6, p. 368-396, 2013. Disponível em: <http://www.scielo.br/pdf/rac/v17n3/a07v17n3.pdf>. Acesso em: 23/04/2016.

TAUCHEN, J.; BRANDLI, L. L. A Gestão Ambiental em Instituições de Ensino Superior: Modelo para Implantação em Campus Universitário. Gestão e produção, v.13, n.3, p. 503-515.2006. Disponível em:

<http://www.scielo.br/pdf/gp/v13n3/11.pdf>. Acesso em: 02/11/2015.

ZANDONAI, D. P.; SAQUETO, K. C.; ABREU, S. C. S. R.; LOPES, A. P.; ZUIN, V. G. Química Verde e Formação de Profissionais do Campo da Química: Relato de uma Experiência Didática para Além do Laboratório de Ensino. Revista. Virtual de Química, vol. 6, n.1, p. 73-84. 2014. Disponível em:

<http://rvq.sbq.org.br/imagebank/pdf/v6n1a07.pdf>. Acesso em: 22/04/2016.

Recebido: 29 mai. 2017

Aprovado: 29 ago. 2017

DOI: 10.3895/actio.v2n2..6812

Como citar:

PAGNO, V.; SALAPATA, A.; SCHMITZ, E. P. S.; CABRERA, L. C. Levantamento de resíduos de

laboratórios, propostas de atividades experimentais e ações com foco em Química Verde. ACTIO, Curitiba, v.

2, n. 2, p. 80-96, jul./set. 2017. Disponível em: <https://periodicos.utfpr.edu.br/actio>. Acesso em: XXX.

Correspondência:

Vanessa Pagno

Avenida Edmundo Gaievski, 1000, Acesso Rodovia PR 182, km 466. Realeza, Paraná, Brasil.

Direito autoral: Este artigo está licenciado sob os termos da Licença CreativeCommons-Atribuição 4.0

Internacional.

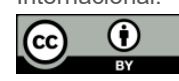

